\title{
Electron capture and emission by the Ti acceptor level in GaP
}

\author{
P. Roura and J. R. Morante \\ Departament de Física Aplicada i Electrònica, Universitat de Barcelona, Av. Diagonal 645, \\ E-08028 Barcelona, Catalonia, Spain \\ G. Guillot and G. Bremond \\ Laboratoire de Physique de la Matière (CNRS URA 358), Bât 502, Institut National des Sciences \\ Appliquées de Lyon, 20 Avenue A. Einstein, 69621 Villeurbanne Cedex, France \\ W. Ulrici \\ Paul Drude Institut für Festkörperelecktronik, Hausvogteiplatz 5-7, 10117 Berlin, Germany
}

(Received 21 March 1995; accepted for publication 24 April 1995)

\begin{abstract}
Previously reported results on deep level optical spectroscopy, optical absorption, deep level transient spectroscopy, photoluminescence excitation, and time resolved photoluminescence are reviewed and discussed in order to know which are the mechanisms involved in electron capture and emission of the Ti acceptor level in GaP. First, the analysis indicates that the ${ }^{3} T_{1}(F)$ crystal-field excited state is not in resonance with the conduction band states. Second, it is shown that both the ${ }^{3} T_{2}$ and ${ }^{3} T_{1}(F)$ excited states do not play any significant role in the process of electron emission and capture. (c) 1995 American Institute of Physics.
\end{abstract}

\section{INTRODUCTION}

Free carrier capture by point defects in semiconductors is accounted for by different mechanisms depending on the defect characteristics. Thus, cascade capture explains correctly the large capture cross sections of hydrogenic impurities. ${ }^{1}$ In this process the carrier losses its energy by dropping through the closely spaced levels that arise from the long range coulombic potential of the impurity. In the case of deep levels, multiphonon emission ${ }^{2}$ and Auger effect ${ }^{3}$ are frequently needed to explain the large amount of energy to be dissipated during the capture.

Although these two mechanisms are the relevant ones for deep levels, there are special situations where cascade capture may contribute as well. In the case where the deep level has a net electrical charge before capture, the coulombic potential implies the existence of a series of hydrogeniclike excited states close to the bands and, as shown in $\mathrm{Si}$ :Se and $\mathrm{Si}: \mathrm{S},{ }^{4}$ their existence may enhance carrier capture. The carrier is captured in a first stage by the hydrogenic-like states and, afterwards it relaxes by multiphonon emission to the deeper ground state. A similar situation can be found when the deep level has excited states of the same nature as the ground state. This is the case for many transition metals in semiconductors. In this situation, the excited states can contribute to carrier capture, but now the energy involved in the capture by the excited state would be relaxed via a multiphonon emission process. On the other hand, these excited states can increase the electron emission by introducing an alternative process that would require thermal excitations with smaller energies [see Fig. 1(a)].

In this article, the electron capture and emission processes of the $\mathrm{Ti}^{2+} / \mathrm{Ti}^{3+}$ acceptor level in $\mathrm{GaP}$ are analyzed. The crystal-field potential splits the $3 d$ atomic levels of the $\mathrm{Ti}^{2+}$ charge state into the ${ }^{3} A_{2}$ ground state and several excited states $\left[{ }^{3} T_{2},{ }^{3} T_{1}(F)\right.$ and $\left.{ }^{3} T_{1}(P)\right]$ [see Fig. 1(b)]. A special attention will be devoted to the possible contribution of the ${ }^{3} T_{2}$ and ${ }^{3} T_{1}(F)$ levels to electron capture and emission.

\section{CAPTURE AND EMISSION THROUGH A MULTIPHONON EMISSION (MPE) PROCESS}

In this section, the expressions governing the capture and emission of free carriers within the framework of the MPE are reviewed. As we want to analyze the influence of the excited states in $3 d$ impurities that arise from the crystal field splitting of atomic $3 d$ levels, the cascade capture pro-. cess can be discarded. On the other hand, Auger processes are only relevant at high carrier concentration. However, under the experimental conditions the carrier concentration was very low. The time decay measurements were done on a semi-insulating sample whereas the emission rates were measured by space charge techniques and correspond to the impurities located at the depleted region where no free carricrs are present. So, only the MPE processes are relevant to analyze the results given in this article.

\section{A. Capture and emission from the ground state}

To analyze MPE processes it is useful the so-called configuration diagram (Fig. 2). ${ }^{5}$ The parabolas represent the adiabatic potential that govern the lattice vibrations around the impurity. A nonradiative transition (capture or emission) takes place at the crossing point between both parabolas. So, an activation energy $\Delta E^{G}$ and $\Delta E^{G}+E_{0}$ is necessary for capture and emission, respectively. Alternatively, the carrier can be promoted to the band with absorption of one photon of energy equal or greater than $E_{0} \cdot{ }^{6}$ That is, $E_{0}$ is the optical threshold corresponding to the absorption band. The optical transition with energy $E_{0}$ is the so-called zero-phonon transition because before and after the transition the vibrational energy is kept at its minimum value. At this point we should clarify a usual misunderstanding. The optical threshold is not given by the vertical transition with energy $E_{n}$ shown in Fig. 1. This energy corresponds simply to the transition with the greatest probability. In the case of an internal transition between the ground and excited state of an impurity, at $E_{n}$ the 
a)

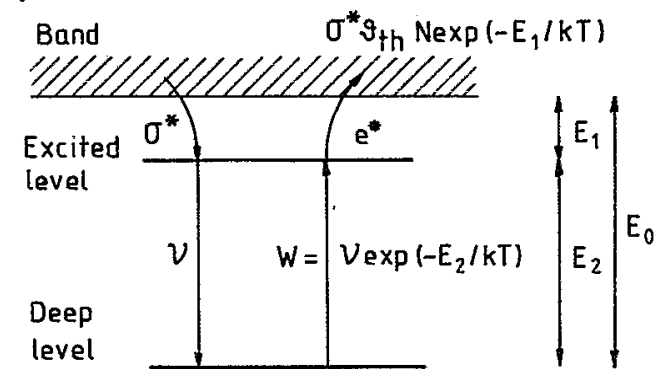

b)

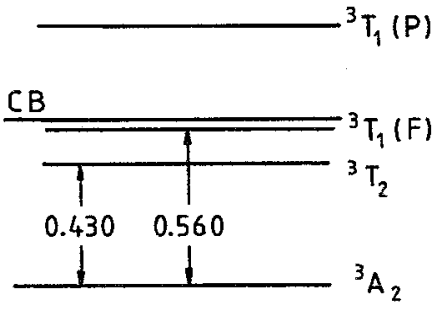

FIG. 1. (a) Parameters involved in carrier emission and capture through an excited state. (b) Energy level structure corresponding to the $\mathrm{Ti}^{2+}$ defect in GaP (energies are in $\mathrm{meV}$ ).

optical absorption spectrum would show a maximum. However, when the conduction or valence bands are involved, there is no special feature related to $E_{n}{ }^{6}$

The capture cross section can be analyzed quantitatively following the classical paper devoted to the MPE capture. ${ }^{2}$ If temperature is high enough, the capture cross section of a deep level can be written as

$$
\sigma^{G}=\sigma_{\infty}^{G} e^{-\Delta E^{G} / k T}
$$

where $\Delta E^{G}$ is the activation energy for the capture (shown in Fig. 2) and $\sigma_{\infty}^{G}$ is the value at the limit of high temperatures. It is found that, for a MPE capture process in III-V semiconductors $\sigma_{\infty}^{G}=10^{-14}-10^{-15} \mathrm{~cm}^{2}$. Detailed balance arguments allow us to calculate the corresponding emission rate

$$
e^{G}=\sigma^{G} v_{\mathrm{th}} N e^{-E_{0} / k T} \propto T^{2} e^{-\left(E_{0}+\Delta E^{G}\right) / k T},
$$

where $v_{\text {th }}$ is the thermal velocity, $N$ the band density of states, and $E_{0}$ the energy difference between the level and the bottom (top) of the conduction (valence) band.

\section{B. Capture and emission via an excited state}

The analysis developed below is taken from the more complete work of Ref. 7. If an excited state is placed at an energy $E_{1}$ below the band [Fig. 1(a)], then it gives an alternative path for carrier capture and emission. When a carrier

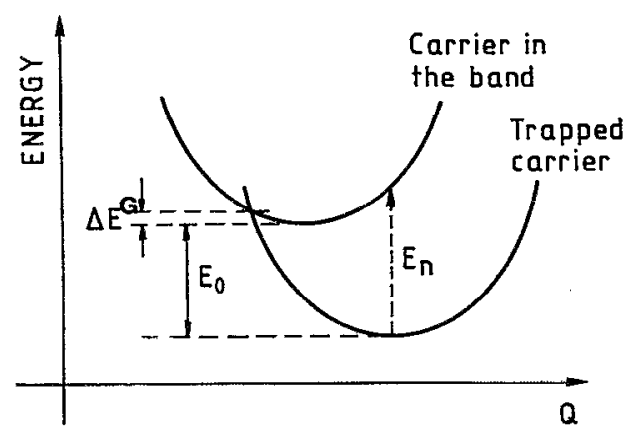

FIG. 2. Electronic plus elastic energies versus lattice coordinate, $Q$. Nonradiative transitions take place at the crossing point. So, an activation energy $\Delta E^{G}$ is needed for capture and $E_{0}+\Delta E^{G}$ for carrier emission. For optical absorption, the most probable transition has an energy $E_{n}$ but the optical threshold is $E_{0}$. is captured by the excited level it can be re-emitted to the band or it can decay to the ground state. Thus, the obscrved capture cross section into the ground state would be

$$
\sigma_{T}^{*}=\frac{\sigma^{*} \nu}{\nu+e^{*}}
$$

where, $\nu$ is the decay probability to the ground state, $e^{*}$ is the emission rate from the excited level to the band

$$
e^{*}=\sigma^{*} v_{\mathrm{th}} N e^{-E_{1} / k T}
$$

and $\sigma^{*}$ is the excited level capture cross section, $v_{\text {th }}$ the thermal velocity, and the $N$ the band density of states. Conversely, in the emission process the carrier promoted to the excited level at a rate

$$
W=\nu e^{-E_{2} / k T}
$$

can decay or can be emitted to the band, giving a rate of thermal emission of

$$
e_{T}^{*}=\frac{W e^{*}}{\nu+e^{*}} .
$$

At high temperatures when thermalization between the band and the excited level is more likely than deep level capture, $e^{*} \gg \nu$, the expressions simplify to

$$
\sigma_{T}^{*} \simeq \frac{\nu e^{E_{\mathrm{l}} / k T}}{v_{\mathrm{th}} N}, \quad e_{T}^{*} \simeq W .
$$

Until now, the expressions are completely general. They do not depend on the mechanism by which the energy is dissipated or absorbed. In the case of MPE processes, $\sigma^{*}$ and $\nu$ are thermally activated according to Refs. 2 and 5

$$
\sigma^{*}=\sigma_{\infty}^{*} e^{-\Delta E^{*} / k T}, \quad \nu=\nu_{\infty} e^{-\Delta E^{\nu / k T}} .
$$

Substitution of (8) into (7) gives the expected dependencies with temperature,

$$
\sigma_{T}^{*} \propto T^{-2} e^{\left(E_{1}-\Delta E^{\nu}\right) / k T}, \quad e_{T}^{*} \propto e^{-\left(E_{2}+\Delta E^{\nu}\right) / k T}
$$

that are to be compared with formulas (1) and (2).

\section{ANALYSIS OF ELECTRON EMISSION BY $\mathrm{Ti}^{2+}$}

The analysis given in the previous section affords a test to know if an excited level contributes to carrier emission by 


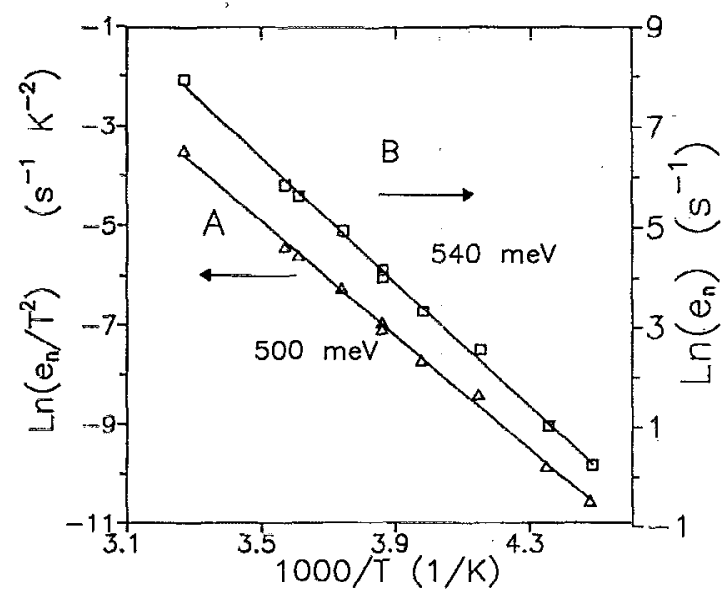

FIG. 3. Electron emission rate of the Ti acceptor level analyzed according to a direct emission to the band $(A)$ and emission through an excited state $(B)$.

comparing the emission rate with the optical spectrum. In the case of a direct emission from the ground state, the activation energy of the emission rate $E_{0}+\Delta E^{G}$ [Eq. (2)] must be greater than the optical absorption threshold, $E_{0}$. Otherwise, a negative value of $\Delta E^{G}$ would be in contradiction with the MPE theory (Fig. 2).

The departure point of this work is an apparent contradiction in the interpretation of results published previously concerning the level structure of the Ti-acceptor center in GaP. In Fig. 1(b) the lowest $\mathrm{Ti}^{2+}$ crystal-field levels are shown. The position of the ${ }^{3} T_{2}$ and the ${ }^{3} T_{1}(F)$ levels relative to the ${ }^{3} A_{2}$ ground state were determined by optical absorption $(\mathrm{OA})^{8}$ and photoluminescence (PL), ${ }^{9}$ respectively. In these previous works the $0.56 \mathrm{eV}$ optical threshold was interpreted as the Ti-acceptor position relative to the conduction band because it was also the threshold of the photoneutralization transition

$$
h \nu+\mathrm{Ti}^{2+} \rightarrow \mathrm{Ti}^{3+}+e_{\mathrm{CB}}
$$

measured by deep level optical spectroscopy (DLOS). ${ }^{10}$ However, as it will be shown below, the acceptor state should be placed deeper in the band gap so that the ${ }^{3} T_{1}(F)$ level is no more resonant with the conduction band states, at least at low temperature.

In Ref. 10, 'deep level transient spectroscopy (DLTS) measurements on the $\mathrm{Ti}$ acceptor level are reported. Its electron emission coefficient, $e_{n}$, is shown in Fig. 3. Within the framework of a direct emission from the ground state, an activation energy of $0.50 \pm 0.02 \mathrm{eV}$ and $\sigma_{\approx}^{G} \approx 1 \times 10^{-15} \mathrm{~cm}^{2}$ are obtained by plotting $e_{n} / T^{2}$ vs $1000 / T$ according to Eq. (2). The activation energy was thought to be the ground state position, $E_{0}$. However, this interpretation is inconsistent with the optical threshold energy of $0.56 \mathrm{eV}$. Substraction of both values delivers a negative activation energy for the capture, $\Delta E^{G}=-60 \mathrm{meV}$. This negative value has no physical meaning within the MPE theory. So, a further analysis is needed.

A priori, we foresee two effects that could explain this discrepancy:

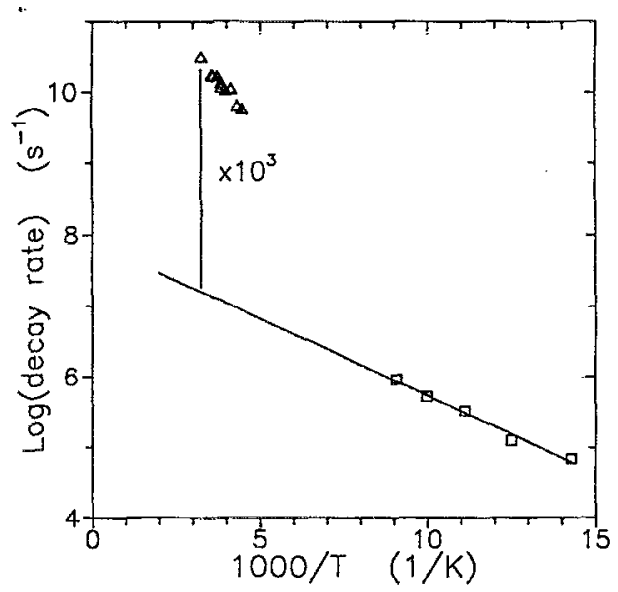

FIG. 4. Internal decay rate of the ${ }^{3} T_{2}$ excited state measured by time resolved PL (squares) compared to the rate needed to account for the electron emission measured by DLTS (triangles).

the possible contribution of the ${ }^{3} T_{1}(F)$ and ${ }^{3} T_{2}$ states to electron and emission and

(ii) the variation of the ground state position $E_{0}$ with temperature.

The second possibility has to be considered because the optical measurements were done at low temperature $(T<100$ $K)$ whereas the electron emission was measured near room temperature. Unfortunately, the evolution of $E_{0}$ is difficult to predict from theoretical considerations. So, we begin with the analysis of the rolc playcd in electron emission and capture by the excited states.

\section{THE ROLE OF ${ }^{3} T_{2}$ EXCITED STATE}

\section{A. Electron emission}

In order to see if there is some contribution of the ${ }^{3} T_{2}$ excited state, let us compare the emission coefficient measured by DLTS (Fig. 3) with the decay rate of this excited state measured by time resolved PL. ${ }^{9}$ By detailed balance arguments we know that the rate of thermal excitation from the ground state, $W$, is related to the decay rate, $\nu$, by Eq. (5) with $E_{2}=430 \mathrm{meV}$. This energy is just the position of the ${ }^{3} T_{2}$ excited state relative to the ground state.

As stated in formula (6) the emission rate via an excited state is smaller than $W$ because it can decay to the ground state before being emitted to the band. So, $W$ gives us an upper limit of the electron emission rate through the ${ }^{3} T_{2}$ level, $e_{T}^{*}$. As we have a direct measurement of the electron emission, $e_{n}$ (Fig. 3), we can compare both values. This has been done in Fig. 4. In fact, in order to have a clearer representation that highlights the experimental errors, we have multiplied $W$ and $e_{n}$ by $e^{430 \mathrm{meV} / k T}$. In this way, $W$ transforms into the decay rate $\nu$ and $e_{n}$ transforms into the decay rate that would be needed to account for the experimental emission rate. Extrapolation of the $\nu$ points indicates that, at room temperature, the contribution of the ${ }^{3} T_{2}$ excited state to electron emission represents less than a $10^{-3}$ part of the total emission rate. As seen in the good alignment of the $\nu$ points, experimental errors cannot substantially affect this conclu- 


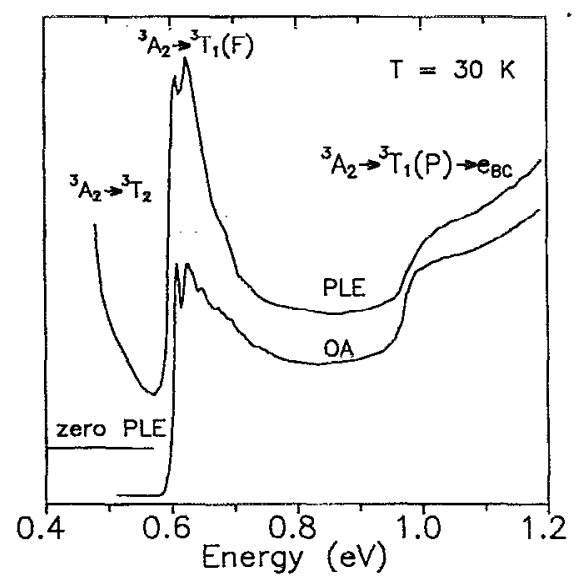

FIG. 5. Optical absorption and photoluminescence excitation spectra measured at low temperature.

sion. This means that thermal excitation from the ${ }^{3} A_{2}$ ground state to the ${ }^{3} T_{2}$ excited state is irrelevant in the electron emission process.

\section{B. Electron capture}

To have an estimation of the ${ }^{3} T_{2}$ excited state contribution to electron capture, we must keep in mind that those electrons being captured by the ${ }^{3} T_{2}$ excited state will emit a photon when decaying to the ground state. That is, the intracenter ${ }^{3} T_{2} \rightarrow{ }^{3} A_{2}$ photoluminescence tells us that electrons are indeed dropping through this excited state. The relevance of this process to the electron capture will be analyzed at low and at room temperatures.

In Fig. 5 the $\mathrm{OA}^{8}$ and the PL excitation (PLE) ${ }^{9}$ spectra at low temperatures are compared. The intracenter transitions to the ${ }^{3} T_{1}(F)$ and ${ }^{3} T_{1}(P)$ states are clearly resolved in both spectra. Due to its high energy, the ${ }^{3} T_{1}(P)$ level is in resonance with the conduction band. In this case, just after intracenter excitation the electron is transferred to the conduction band as indicated in the figure. In the PLE spectrum an extra band appears that corresponds to the symmetry forbidden spin allowed ${ }^{3} A_{2} \rightarrow{ }^{3} T_{2}$ transition. This transition promotes the electron directly into the ${ }^{3} T_{2}$ luminescent level.

The OA and the PLE spectral shapes can be related as follows. The OA coefficient is just the probability one electron in the ground state can be excited by absorption of one photon. This electron will contribute to the PL emission if, when decaying to the ground state, it passes through the ${ }^{3} T_{2}$ level. This process will have a probability, $\eta$, that would depend on the departure state of the excited electron before the decay. So, we can write

$$
I_{\mathrm{PLE}}=A \eta I_{\mathrm{OA}}(h \nu) \text {, }
$$

where $A$ is a constant that accounts for the fact that usually it is impossible to obtain an absolute value of the PLE efficiency. According to the definition given above, $\eta=1$ for the ${ }^{3} A_{2} \rightarrow{ }^{3} T_{2}$ transition whereas

$$
\eta=\sigma^{*}\left({ }^{3} T_{2}\right) / \sigma_{n}
$$

for the transitions leaving an electron in the conduction band. Here, $\sigma^{*}\left({ }^{3} T_{2}\right)$ is the capture cross section of the ${ }^{3} T_{2}$ excited level and $\sigma_{n}$, the overall capture cross section of the Ti acceptor state. If we represented both spectra with the same intensity for the ${ }^{3} A_{2} \rightarrow{ }^{3} T_{2}$ transition $(\eta=1)$, then $A$ would be the unity and comparison of PLE and OA spectra would give a very conservative estimation of $\sigma^{*}\left({ }^{3} T_{2}\right)$,

$$
\sigma^{*}\left({ }^{3} T_{2}\right) / \sigma_{n}<10^{-1} \text {. }
$$

This means that, at low temperature, the contribution to capture of the ${ }^{3} T_{2}$ level is small.

At room temperature, an electron captured by the ${ }^{3} T_{2}$ level can decay to the ground state being effectively captured or it can be re-emitted to the conduction band. We can calculate the decay probability by extrapolation. This has already been done in Fig. 4. The result is $\nu(300 \mathrm{~K})=2.0 \times 10^{7}$ $\mathrm{s}^{-1}$. The re-emission rate can be estimated from Eq. (4). We take for $\sigma_{\infty}^{*}$ the typical value expected for a MPE process, $\sigma_{\infty}^{*}=10^{-15} \mathrm{~cm}^{2}$ and for the $E_{1}$ energy, just the difference between the photoneutralization threshold energy $(560 \mathrm{meV})$ and the ${ }^{3} T_{2}$ level position ( $430 \mathrm{meV}$ ). This calculation gives a value of $e^{*}=2.8 \times 10^{11} \mathrm{~s}^{-1}$ which is about four orders of magnitude greater than the decay rate. So, at room temperature the ${ }^{3} T_{2}$ level cannot contribute appreciably to capture because an electron captured by this level would be reemitted to the conduction band before decaying to the ground state.

\section{THE ROLE OF THE ${ }^{3} T_{1}(F)$ EXCITED STATE}

\section{A. Electron capture}

Contrary to the previous case, the ${ }^{3} T_{1}(F) \rightarrow{ }^{3} A_{2}$ transition has not been observed by PL. So, we do not have any direct measurement that would reflect the capture of electrons through the ${ }^{3} T_{1}(F)$ excited state. In spite of that, Гig. 5 can afford useful information. Apart from the previous discussion on the appearance of the ${ }^{3} A_{2} \rightarrow{ }^{3} T_{2}$ band in the PI.E spectrum, there is a great increase in the intensity of the ${ }^{3} A_{2} \rightarrow{ }^{3} T_{1}(F)$ band. This is in clear contradiction to the level structure deduced in previous works ${ }^{10}$ where the ${ }^{3} T_{1}(F)$ level was supposed to be in resonance with the conduction band. If this were the situation, an internal excitation to ${ }^{3} T_{1}(F)$ will finally leave an electron in the conduction band before capture. So, the probability of reaching the ${ }^{3} T_{2}$ luminescent state [ $\eta$ parameter in Eq. (11)] would be the same than in the higher ${ }^{3} A_{2} \rightarrow{ }^{3} T_{1}(P)$ transition. The fact that $\eta$ is different in the ${ }^{3} A_{2} \rightarrow{ }^{3} T_{1}(F)$ transition indicates that the ${ }^{3} T_{1}(F)$ level is not in resonance with the conduction band but, instead, it is located below it.

In spite of this conclusion one could think that, even if the ${ }^{3} T_{1}(F)$ level were resonant with the conduction band, the intracenter de-excitation could be faster than the electron transfer to the continuum. In this way, the higher intensity of the ${ }^{3} A_{2} \rightarrow{ }^{3} T_{1}(F)$ band in the PLE spectrum could be explained as well. However, there is no experimental evidence of similar intracenter transitions involving a resonant excited level in $3 d$ impurities. If for a particular impurity the optical transition were symmetry allowed, one would expect a radiative emission. However, to our knowledge, no PL band re- 
TABLE I. Decay rates for the first excited state of several transition metal ions in III-V compounds at $300 \mathrm{~K}$.

\begin{tabular}{cccc}
\hline Defect & Transition & $\nu\left(\mathrm{s}^{-1}\right)$ & Ref. \\
\hline $\mathrm{GaAs}: \mathrm{V}^{3+}$ & ${ }^{3} T_{2}-{ }^{3} A_{2}$ & $2.1 \times 10^{5}$ & 11 \\
$\mathrm{GaP}: \mathrm{V}^{3+}$ & ${ }^{3} T_{2}-{ }^{3} A_{2}$ & $1.4 \times 10^{8}$ & 11 \\
$\mathrm{GaP}: \mathrm{Ti}^{3+}$ & ${ }^{2} T_{2}-{ }^{2} E$ & $1.0 \times 10^{6}$ & 12 \\
$\mathrm{InP}: \mathrm{Fe}^{2+}$ & ${ }^{5} T_{2}-{ }^{5} E$ & $8.3 \times 10^{6}$ & 13 \\
$\mathrm{GaP}: \mathrm{Ti}^{2+}$ & ${ }^{3} T_{2}-{ }^{3} A_{2}$ & $1.7 \times 10^{7}$ & This work \\
\hline
\end{tabular}

lated to any excited resonant level has never been reported in the literature for any $3 d$ impurity. On the other hand, these kinds of transitions would be temperature dependent and, consequently, the intensity of the corresponding photoexcitation band would change with temperature. This has never been observed in the great number of resonant levels related to $3 d$ impurities.

\section{B. Electron emlssion}

If the electrons were emitted through the ${ }^{3} T_{1}(F)$ excited state at a temperature high enough so that $e^{*}>\nu$, the emission coefficient would be thermally activated following Eq. (5). An Arrhenius plot of $\log \left(e_{n}\right)$ vs $1000 / T$ would deliver an activation energy close to the excited state energy $E_{2}$. This has been done in Fig. 3 and an activation energy of $540 \pm 15 \mathrm{meV}$ is obtained. This is very similar to the ${ }^{3} T_{1}(F)$ energy relative to the ground state, $560 \mathrm{meV}$. Although one would be tempted to conclude that this is proof that electrons are indeed emitted through the ${ }^{3} T_{1}(F)$ level, it will be shown that this is not the case.

Contrary to the analysis of the ${ }^{3} T_{2}$ level we do not have independent determinations of $e_{T}$ and $W$ to directly compare their values as was done in Fig. 4. Alternatively, data from other $3 d$ transition metals in III-V semiconductors are analyzed. ${ }^{11-13}$ The relevant information is summarized in Table I. The second column indicates the levels involved in the internal deexcitation from the first excited state to the ground state. In all cases this process has been studied by time resolved PL. The decay rate at $300 \mathrm{~K}, \nu$ (third column), has been measured directly or has been extrapolated from the data given by the authors.

We can calculate the value of $\nu$ at $300 \mathrm{~K}$ for the ${ }^{3} T_{1}(F)$ level of $\mathrm{Ti}^{2+}$ in $\mathrm{GaP}$ that would give an excitation probability just equal to the emission rate $e_{n}$ measured by DLTS. Use of Eq. (5) with $E_{2}=0.560 \mathrm{eV}$, gives $\nu\left[^{3} T_{1}(F)\right]=6.3 \times 10^{12} \mathrm{~s}^{-1}$. This value is four orders of magnitude greater that the quickest de-excitation rate tabulated in Table I. So, although we do not have any direct measurement of $\nu$ for the ${ }^{3} T_{1}(F)$ level, we can be sure that it is much smaller than the value needed to account for the electron emission.

\section{INFLUENCE OF THE BAND GAP VARIATION WITH TEMPERATURE}

Now, as we have demonstrated that the excited states of $\mathrm{Ti}^{2+}$ do not contribute appreciably to electron emission, the initial contradiction between the activation energy of $e_{n}$
$(0.50 \mathrm{eV})$ and the optical threshold $(0.56 \mathrm{eV})$ remains. In fact, the energy difference is probably due just to the temperature at which measurements have been done. The OA spectrum of Fig. 5 was measured at $30 \mathrm{~K}$, whereas the temperature of the emission rate in Fig. 3 ranges from 220 to $300 \mathrm{~K}$.

The evolution of the ionization energy with temperature has been measured for other impurities and there are no general rules to predict it. As an example, we can look at the $O$ level in $\mathrm{GaP} .{ }^{14}$ Below $175 \mathrm{~K}$ the level is pinned to the valence band, whereas at higher temperatures the diminution of the band gap energy is shared by both the conduction and the valence bands.

The variation of the GaP band gap energy is given by the Varshni formula, ${ }^{15}$

$$
E_{g}(T)-E_{g}(0)=-\frac{\alpha T^{2}}{T+\beta},
$$

where $\alpha=5.771 \times 10^{-4} \mathrm{eV} \mathrm{K}^{-2}$ and $\beta=372 \mathrm{~K}^{16}$ From $30 \mathrm{~K}$ until $250 \mathrm{~K}$ the band gap energy diminishes by $57 \mathrm{meV}$. If the Ti acceptor level were pinned to the valence band, then the ionization energy would be reduced by this amount and the apparent contradiction would disappear.

This leads us to a quite unusual situation for the $\mathrm{Ti}^{2+} / \mathrm{Ti}^{3+}$ acceptor level. At room temperature, its ground state would be placed at $0.50 \mathrm{eV}$ below the conduction band, whereas at low temperature this energy would increase to somewhat more than $560 \mathrm{meV}$, so that the ${ }^{3} T_{1}(F)$ level would be no more resonant with the conduction band as shown in Fig. 2(b).

\section{CONCLUSIONS}

Comparison of a number of previously published results leads to the conclusion that, in the case of $\mathrm{Ti}^{2+}$ in GaP, its $3 d$ crystal-field excited levels do not contribute appreciably to electron capture and emission. The emission rate through the ${ }^{3} T_{2}$ level has been calculated by using detailed balance arguments. The result gives a value that is about $10^{-3}$ times the total emission rate. In the case of the ${ }^{3} T_{1}(F)$ level, its contribution to electron emission is analyzed in view of PL decay lifetimes of other $3 d$ impurities in III-V semiconductors. From a comparison between OA and PLE spectra it has been concluded that, at low temperatures, the ${ }^{3} T_{1}(F)$ level is not resonant with the conduction band, contrary to the previous model for the Ti acceptor level. ${ }^{8}$ This conclusion should be tested by DLOS measurements done at low temperature. The photoneutralization band corresponding to this level (Fig. 5) should be thermally activated.

\footnotetext{
${ }^{1}$ M. Lax, Phys. Rev. 119, 150 (1960).

${ }^{2}$ C. H. Henry and D. V. Lang, Phys. Rev. B 15, 989 (1977).

${ }^{3}$ T. Landsberg, Phys. Status Solidi 41, 457 (1970).

${ }^{4}$ G. R. Rees, H. G. Grimmeiss, E. Janzen, and B. Skarstam, J. Phys. C: Solid State Phys. 13, 6157 (1980).

${ }^{5}$ K. K. Rebane, Impurity Spectra of Solids (Plenum, New York, 1970).

${ }^{\circ}$ B. Monemar and L. Samuelson, Phys. Rev. B 18, 809 (1978).

${ }^{7}$ R. Gibb, G. J. Rees, B. W. Thomas, B. L. M. Wilson, B. Hamilton, D. R. Wight, and N. F. Mott, Philos. Mag. 6, 1021 (1977).
} 
${ }^{8}$ W. Ulrici, K. Friedland, L. Eaves, D. P. Halliday, and L. A. Payling, Phys. Status Solidi B 150, 177 (1988).

${ }^{9}$ P. Roura, T. Benyattou, G. Guillot, R. Moncorge, and W. Ulrici, Semicond. Sci. Technol. 4, 943 (1989).

${ }^{10}$ P. Roura, G. Bremond, A. Nouailhat, G. Guillot, and W. Ulrici, Appl. Phys. Lett. 51, 1696 (1987).

${ }^{11}$ C. Benjeddou, J. H. Crasemann, G. Guillot, C. Charreaux, A. Nouailhat, and H.-J. Shulz, J. Phys. C: Solid State Phys. 20, 2789 (1987).
${ }^{12}$ P. Roura, T. Benyattou, G. Guillot, R. Moncorge, and W. Ulrici, Phys. Rev. B 45, 11698 (1992).

${ }^{13}$ P. B. Klein, J. E. Furneaux, and R. L. Henry, Phys. Rev. B 29, 1947 (1984).

${ }^{14}$ L. Samuelson and B. Monemar, Phys. Rev, B 18, 830 (1978).

${ }^{15}$ Y. P. Varshni, Physica 34, 149 (1967).

${ }^{16}$ C. D. Thurmond, J. Electrochem. Soc. 122, 1133 (1975). 